\title{
ON THE GROWTH RATE OF CONTRACTIBLE CLOSED GEODESICS ON REDUCIBLE MANIFOLDS
}

\author{
GABRIEL P. PATERNAIN AND JIMMY PETEAN
}

\begin{abstract}
We prove exponential growth rate of contractible closed geodesics for an arbitrary bumpy metric on manifolds of the form $X_{1} \# X_{2}$ where the fundamental group of $X_{1}$ has a subgroup of finite index at least 3 and $X_{2}$ is simply connected and not a homotopy sphere.
\end{abstract}

\section{INTRODUCTION}

Let $M$ be a closed manifold and $g$ a smooth Riemannian metric on $M$. Given $t \geq 0$, define $N_{g}(t)$ to be the number of geometrically distinct closed geodesics of $g$ with length $\leq t$. Similarly, let $N_{g}^{0}(t)$ be the number of geometrically distinct contractible closed geodesics of $g$ with length $\leq t$.

If $g$ is a bumpy metric, i.e., all the closed geodesics of $g$ are non-degenerate, then $N_{g}(t)$ is finite for each $t$ and we can ask the following basic question: what is the behaviour of $N_{g}(t)$ as $t$ tends to infinity? Similarly if $g$ is a 0 -bumpy metric, i.e., all the contractible closed geodesics of $g$ are non-degenerate, then $N_{g}^{0}(t)$ is finite for each $t$ and we can also wonder about its growth.

Let $\Lambda(M)$ be the space of piecewise differentiable closed curves $c: \mathbb{R} / \mathbb{Z} \rightarrow M$, endowed with the compact-open topology.

Proposition A. Let $M$ be a closed manifold and let $X \subset M$ be a simply connected submanifold, possibly with boundary. Consider the inclusion map $\iota: X \rightarrow M$ and let $R_{i}$ be the rank of the map induced in the loop space homology for some field of coefficients $k_{p}, p$ prime or zero. Then, for any 0-bumpy metric $g$ on $M$ there exist constants $\alpha=\alpha(g)>0$ and $\beta=\beta(g)>0$ such that

$$
N_{g}^{0}(t) \geq \alpha \max _{i \leq \beta t} R_{i}
$$

for all t sufficiently large.

This proposition is a generalization of a theorem of M. Gromov [11, later improved by W. Ballmann and W. Ziller [6], who proved the proposition when $M$ is simply connected and $X=M$ (we remark that in Gromov's theorem it is essential to assume that $M$ is simply connected). In fact, the methods in 22 show that the proposition is still true if we replace $X$ by an arbitrary finite simply connected CW-complex $K$ and $\iota$ by any continuous map $f: K \rightarrow M$.

Date: May 2004.

G. P. Paternain was partially supported by CIMAT, Guanajuato, México.

J. Petean is supported by grant $37558-\mathrm{E}$ of CONACYT. 
The result has the following interesting consequence:

Theorem B. Let $M$ be a closed manifold of dimension $n \geq 3$. Suppose that $M$ can be decomposed as $X_{1} \# X_{2}$, where $\pi_{1}\left(X_{1}\right)$ has a subgroup of finite index $\geq 3$ and $X_{2}$ is simply connected. Then, for any 0-bumpy metric $g$ on $M, N_{g}^{0}(t)$ grows exponentially with $t$ unless $X_{2}$ is a homotopy sphere.

The novelty in Theorem $\mathrm{B}$ lies in the fact that $M$ is allowed to have any fundamental group as long as it has subgroups of finite index $\geq 3$. Results of this kind, when $M$ is simply connected were obtained by P. Lambrechts in [18] and in fact, we will rely quite heavily on his Hochschild homology computations.

We note that there are finitely presented infinite groups without proper subgroups of finite index. An example is given by the Higman 4-group, see [23]. We do not know if Theorem B is still true for such groups.

A celebrated theorem of D. Gromoll and W. Meyer [10] asserts that any Riemannian metric on a closed simply connected manifold $M$ has infinitely many geometrically distinct closed geodesics if the Betti numbers of $\Lambda(M)$ are unbounded for some field of coefficients. Their methods combined with our proof of Theorem B yield the existence of infinitely many geometrically distinct contractible closed geodesics for any metric on a manifold as in Theorem B. We do not know if the theorem is still true if we drop the bumpy condition and we just assume $N_{g}^{0}(t)$ finite for all $t$.

There are several papers establishing lower bounds for the growth of $N_{g}(t)$ in the presence of fundamental group [4, 5, 17, 8, 15]. These bounds are not exponential (e.g. $t / \log t)$ and do not give information about contractible closed geodesics, but they do hold for any Riemannian metric.

The present note is a spin-off of our investigations on the topological entropy $\mathrm{h}_{\text {top }}(g)$ of the geodesic flow [20, 21. Except in the case of surfaces, there is basically no relationship between positivity of $\mathrm{h}_{\text {top }}(g)$ and exponential growth for $N_{g}(t)$. M. Herman gave in [14] an example of a minimal real analytic diffeomorphism on a closed 4manifold with positive topological entropy. However, if the geodesic flow of $g$ has a horseshoe, then the number of hyperbolic closed geodesics of $g$ grows exponentially. It has been conjectured that generically in the $C^{k}$ topology, $2 \leq k \leq \infty$, the geodesic flow of $g$ has a horseshoe, but this has only been proved for surfaces [9, 17.

Acknowledgements: We thank Wolfgang Ziller and Werner Ballmann for various comments on the first draft of the manuscript.

\section{A BRIEF REVIEW OF MORSE THEORY OF THE LOOP SPACE}

Let $\Lambda=\Lambda(M)$ be the space of piecewise differentiable closed curves $c: S^{1}=$ $\mathbb{R} / \mathbb{Z} \rightarrow M$, endowed with the compact-open topology, i.e. the topology induced by the metric $\rho\left(c, c^{\prime}\right)=\max _{t \in S^{1}} d\left(c(t), c^{\prime}(t)\right)$. The energy functional $E: \Lambda \rightarrow \mathbb{R}$ is defined

by $E(c)=\frac{1}{2} \int_{0}^{1}\langle\dot{c}(t), \dot{c}(t)\rangle d t$. The critical points of $E$ are the closed geodesics and the point curves. If $\ell(c)$ denotes the length of $c$, then the Cauchy-Schwarz inequality implies that $\ell(c)^{2} \leq 2 E(c)$ with equality if and only if $c$ is parametrized proportional to arc length. 
There is a natural action of $S^{1}$ on $\Lambda$. Given $s \in S^{1}$ and $c \in \Lambda$, we can define $s c$ by $s c(t):=c(t+s)$. If $c$ does not reduce to a point, then the isotropy group of $c$ is a finite subgroup of $S^{1}$ isomorphic to $\mathbb{Z}_{k}$ for some $k \geq 1$. In this case, $k$ is called the multiplicity of $c$ and we can write $c=d^{k}$, where $d^{k}(t)=d(k t)$. The curve $c$ is called the $k$-th iterate of $d$.

If $c$ is a closed geodesic, then the entire orbit of $c$ under the $S^{1}$-action is a set of critical points of $E$. A closed geodesic $c$ is said to be non-degenerate if the orbit of $c$ is a non-degenerate critical submanifold of $\Lambda$. Equivalently, $c$ is non-degenerate if and only if there is no periodic Jacobi field along $c$ orthogonal to $\dot{c}$. In more dynamical terms, $c$ is non-degenerate if and only if the linearized Poincaré map of the orbit of the geodesic flow corresponding to $c$ does not have 1 as an eigenvalue.

A metric is said to be bumpy (resp. 0-bumpy) if all closed (resp. contractible) geodesics are non-degenerate. The bumpy metric theorem asserts that the set of $C^{r}$ bumpy metrics is a residual subset of the set of all $C^{r}$ metrics endowed with the $C^{r}$ topology for all $2 \leq r \leq \infty$. The bumpy metric theorem is traditionally attributed to R. Abraham [1, but see also Anosov [2] and Klingenberg and Takens [16]. Obviously bumpy implies 0-bumpy.

\section{Proof of Proposition A}

We will need the following version of a result due to M. Gromov 11] for manifolds with non-empty boundary, see also [12, p. 102], 13, 3, 19. In all these references the manifold is assumed to have empty boundary. In the case of the pointed loop space $\Omega(X)$ we have written down a proof of the theorem for compact manifolds with non-empty boundary in [21. For completeness we will show in the appendix that the details work as well for the free loop space $\Lambda(X)$.

Theorem 3.1. Given a metric $g$ on a simply connected compact manifold $X$ (possibly with boundary), there exists a constant $C=C(g)>0$ such that any element in $H_{i}\left(\Lambda(X), k_{p}\right)$ can be represented by a cycle whose image lies in $\Lambda^{C i}(X)$.

Let us prove now Proposition A. Let $\Lambda_{0} \subset \Lambda$ denote the subspace of contractible closed loops. If all the contractible closed geodesics are non-degenerate, then given $a>0$, there exist only finitely many such closed geodesics $c_{1}, \ldots, c_{r}$ with energy precisely $a$. There also exists an $\varepsilon>0$ such that there is no contractible closed geodesic $c$ with $E(c) \neq a$ but $a-\varepsilon \leq E(c) \leq a+\varepsilon$. It follows from Morse theory that $\Lambda_{0}^{a+\varepsilon}$ is homotopy equivalent to $\Lambda_{0}^{a-\varepsilon}$ with cells $e_{i}, \bar{e}_{i}$ attached, where $\operatorname{dim} e_{i}=$ $\operatorname{dim} \bar{e}_{i}-1=\operatorname{index}$ of $c_{i}$. It follows from this that $\left(\Lambda_{0}^{a}, M\right)$ is homotopy equivalent to a relative CW-complex, where the number of $k$-cells attached to $M$ is equal to the number of critical circles $S^{1} c$ of $E$ with index of $c$ equal to $k$ or $k-1$ and $0<E(c) \leq a$. Observe that the iterates of a closed geodesic may give rise to different critical circles of $E$ of index $k$ or $k-1$.

Let $b_{i}(t):=\operatorname{dim} H_{i}\left(\Lambda_{0}^{t}, M\right)$. It follows from the above that the number of prime and iterate contractible closed geodesics of index $i$ or $i-1$ and length $\leq t$ is $\geq b_{i}(t)$.

We will need the following lemma proved in [6] that gives control of the contribution of a closed geodesic $c$ and its iterates to $b_{i}(t)$. The lemma is not properly stated as 
such in [6], but the statement can be found at the end of the proof of their main theorem.

Lemma 3.2. There exists a constant $n_{0}=n_{0}(g)$ such that at most $n_{0}$ iterates of a contractible closed geodesic of length $\leq t$ can have index $i$ or $i-1$ if $t / 2<i \leq t$.

From the lemma it follows that $N_{g}^{0}(t) \geq \max _{t / 2<i \leq t} b_{i}(t) / n_{0}$. Since $N_{g}^{0}(t) \geq N_{g}^{0}(t / 2)$ we obtain $N_{g}^{0}(t) \geq \max _{1<i \leq t} b_{i}(t) / n_{0}$.

Proposition $\mathrm{A}$ is now a consequence of the following lemma:

Lemma 3.3. There exists a constant $C=C(g)>0$ such that $b_{i}(t) \geq R_{i}$ for $n+1<$ $i \leq C t$.

Proof. First note that $H_{i}\left(\Lambda_{0}^{t}\right) \cong H_{i}\left(\Lambda_{0}^{t}, M\right)$ for $i>n+1$, so we only need to prove that $b_{i}\left(\Lambda_{0}^{t}\right) \geq R_{i}$ for $i \leq C t$.

Now take an element $e \in H_{i}(\Lambda(X))$. By Theorem 3.1 (we consider $X \subset M$ with the induced metric) there exists $C>0$ (depending on $g$ ) such that whenever $C t \geq i$, there exists a cycle $\eta \in \Lambda^{t}(X)$ such that $\eta$ represents $e$. Since $\iota$ preserves energy, $\iota \circ \eta$ is a cycle in $\Lambda_{0}^{t}$ and the lemma follows.

\section{Proof of Theorem B}

Proof. Let $\widetilde{X_{1}}$ be a finite covering of $X_{1}$ of degree $k \geq 3$. Consider the covering $N$ of $M=X_{1} \# X_{2}$ given by the connected sum of $\widetilde{X_{1}}$ with $k$ copies of $X_{2}$. Since the exponential growth rate of closed geodesics is invariant under finite coverings, we only need to work with $N$. We now want to find a submanifold of $N$ to play the role of $X$ in Proposition A. If $D$ is an $n$-dimensional disc then $X:=D \# 3 X_{2}$ appears as a submanifold of $N$.

Lemma 4.1. The inclusion map $\iota: X \rightarrow N$ induces a map

$$
\iota_{*}: H_{*}\left(\Lambda(X), k_{p}\right) \rightarrow H_{*}\left(\Lambda(N), k_{p}\right)
$$

whose rank grows exponentially for some $p$.

Proof. There is a map $p: M \# 3 X_{2} \rightarrow 3 X_{2}$ obtained by collapsing the exterior and the boundary of a disc $D \subset M$ (which contains the 3 points where the connected sums are taken) to a point. If $j=p \circ \iota$ it is enough to prove that the rank of the map induced by $j$ in the free loop space homology grows exponentially.

Clearly, $j$ is just the inclusion of $D \# 3 X_{2}$ in $3 X_{2}=S^{n} \# 3 X_{2}$. In particular, the computations done in [18] apply to this problem. If $X_{2}$ is not a homotopy sphere, there is a minimal degree in which its homology is non-trivial for some coefficient field $k_{p}$. In $3 X_{2}$ we have then three cohomology classes in this minimal degree which are independent and whose cup product vanish. These cohomology classes imply the existence of elements in the corresponding DGA's which satisfy the hypothesis of [18, Proposition 9]. Of course, $j$ sends these cohomology classes to corresponding ones in $3 X_{2}-D$. Then one looks at the map induced in Hochschild homology and the exponential growth of the rank follows. 
Theorem B follows directly from the previous lemma and Proposition A.

\section{Appendix: Proof of Theorem 3.1 when $\partial X$ is NON-Empty}

Proof. : We will only modify the proof in 21] to show that it works in the case of the free loop space. Assume that the boundary of $X$ is non-empty. We have a collar of the boundary diffeomorphic to $\partial X \times[0,2)$. Let $Y$ be the manifold obtained by deleting $\partial X \times[0,1)$ from $X$. Of course, $Y$ is diffeomorphic to $X$. Now find a finite number of convex subsets of $X$ which cover $Y$. Call them $V_{\alpha}, 1 \leq \alpha \leq k_{0}$. Let $\mathbf{T}$ be a triangulation of $Y$. For a point $p \in Y$, let $F(p)$ be the closed cell of lowest dimension containing $p$ and let $O(p)$ be the union of all closed cells intersecting $F(p)$. Note that $O(p)$ is a compact subset of $Y$. Similarly, for any subset $K \subset Y$ one can define $F(K)$ as the union of $F(p)$ for $p \in K$ and $O(K)$ as the union of $O(p)$ for $p \in K$. There is a positive number $\delta$ (depending on the covering $\left\{V_{\alpha}\right\}$ and $g$ ) such that, after taking some barycentric subdivisions, we can take $\mathbf{T}$ so that for every subset $K$ of diameter bounded by $\delta, O(K)$ is contained in one of the $V_{\alpha}$ 's.

Now we define the open subset $\Lambda_{k}$ of $\Lambda\left(\cup V_{\alpha}\right)$ as the set of all paths $\omega$ in $\cup V_{\alpha}$ with $\omega(0)=\omega(1)$ so that for all $j$ between 1 and $2^{k}$,

$$
O\left(\left\{\omega\left(j-1 / 2^{k}\right), \omega\left(j / 2^{k}\right)\right\}\right) \cup \omega\left[j-1 / 2^{k}, j / 2^{k}\right]
$$

is contained in one of the $V_{\alpha}$ 's. It is easy to see that $\Lambda(Y)$ is contained in the union of the $\Lambda_{k}$ 's.

Let $B_{k}$ be the set of sequences $p_{0}, \ldots, p_{2^{k}}$ of points in $Y$ such that $p_{0}=p_{2^{k}}$ and for each $j$ between 1 and $2^{k} O\left(\left\{p_{j-1}, p_{j}\right\}\right)$ is contained in one of the $V_{\alpha}$ 's. Let $\Lambda_{k}^{Y} \subset \Lambda_{k}$ be the set of paths in $\Lambda_{k}$ for which all $2^{k}$ points $\omega\left(j / 2^{k}\right)$ are in $Y$. Then $B_{k}$ is naturally identified with a subset of $\Lambda_{k}^{Y}$ (an element of $B_{k}$ uniquely determines a broken geodesic which sends $j / 2^{k}$ to $p_{j}$ ) and it is actually a deformation retract of $\Lambda_{k}^{Y}$.

Given a cycle representing a homology class in $\Lambda(X)$ it can be represented by a cycle in $\Lambda(Y)$ which is therefore contained in $\Lambda_{k}^{Y}$ for some $k$. Therefore we can retract it to $B_{k}$. But $B_{k}$ is easily identified with a subset of $Y^{2^{k}}$. Moreover, under this identification if a point $\left(p_{1}, \ldots, p_{2^{k}}\right) \in B_{k}$, then the whole $F\left(p_{1}\right) \times \ldots \times F\left(p_{2^{k}}\right)$ is contained in $B_{k}$. This implies that $\mathbf{T}$ induces a cell decomposition in $B_{k}$. Hence the $i$-homology class can be represented by a combination of cells of dimension $i$. A cell in $B_{k}$ is a product of cells in each coordinate. The dimension of such a cell is the sum of the dimensions of the corresponding cells, of course. If the total dimension is $i$ then there can be at most $i$ cells of positive dimension. Since $X$ is simply connected there exists a smooth map $f: X \rightarrow X$ which is smoothly homotopic to the identity and which sends the union of the images of all the geodesic segments joining vertices in the triangulation to a point. The norm of the differential of $f$ is bounded since $X$ is compact and $f$ induces a map $\hat{f}: \Lambda(X) \rightarrow \Lambda(X)$ which is homotopic to the identity. Now paths belonging to an $i$-cell of $B_{k}$ are formed by pieces joining vertices of the triangulation and at most $2 i$ pieces in which one of the points is not a vertex. Under

the map $\hat{f}$ the former are sent to a point and the latter to a path of length bounded 
in terms of the norm of the differential of $f$ and the diameter of the $V_{\alpha}$ 's. Therefore, there exists a constant $C(g)$ such that the image of the $i$-skeleton of $B_{k}$ is sent by $\hat{f}$ to the subset of paths with energy bounded by $C(g) i$. The theorem follows.

\section{REFERENCES}

[1] R. Abraham, Bumpy metrics, Global Analysis, Proc. Sympos. Pure Math. vol. XIV (S.S. Chern and S. Smale, eds.), 1970, pp. 1-3.

[2] D.V. Anosov, On generic properties of closed geodesics, Math. USSR, Izv. 21 (1983), 1-29.

[3] I. Babenko, Topological entropy of geodesic flows on simply connected manifolds, and related problems, (Russian) Izv. Ross. Akad. Nauk. Ser. Mat. 61 (1997) 57-74.

[4] W. Ballmann, Gescholssene Geodätische auf Mannigfaltigkeiten mit unendlicher Fundamentalgruppe, Topology 25 (1986) 55-69.

[5] W. Ballmann, G. Thorbergsson, W. Ziller, Closed geodesics and the fundamental group, Duke Math. J. 48 (1981) 585-588.

[6] W. Ballmann, W. Ziller, On the number of closed geodesics of a compact Riemannian manifold, Duke Math. J. 49 (1982) 629-632.

[7] V. Bangert, N. Hingston, Closed geodesics on manifolds with infinite abelian fundamental group, J. Differential Geom. 19 (1984) 277-282.

[8] V. Bangert, W. Klingenberg, Homology generated by iterated closed geodesics, Topology 22 (1983) 379-388.

[9] G. Contreras, G.P. Paternain, Genericity of geodesic flows with positive topological entropy on $S^{2}$, J. Differential Geom. 61 (2002) 1-49.

[10] D. Gromoll, W. Meyer, Periodic geodesics on compact Riemannian manifolds, J. Differential Geom. 3 (1969) 493-510.

[11] M. Gromov, Homotopical effects of dilatations, J. Diff. Geom. 13 (1978) 303-310.

[12] M. Gromov, Structures métriques pour les variétés Riemanniennes, by J. Lafontaine and P. Pansu, Editions Cedic/Fernand Nathan, Paris, 1981.

[13] M. Gromov, Metric structures for Riemannian and non-Riemannian spaces, Progress in Mathematics, 152, S.M. Bates (translator) 1999.

[14] M.R. Herman, Construction d'un diffomorphisme minimal d'entropie topologique non nulle, Ergodic Theory Dynamical Systems 1 (1981) 65-76.

[15] N. Hingston, On the growth of the number of closed geodesics on the two-sphere, Internat. Math. Res. Notices 9 (1993) 253-262.

[16] W. Klingenberg, F. Takens, Generic properties of geodesic flows, Math. Ann. 197 (1972) 323334.

[17] G. Knieper, H. Weiss, Genericity of positive topological entropy for geodesic flows on $S^{2}$, J. Differential Geom. 62 (2002) 127-141.

[18] P. Lambrechts, The Betti numbers of the free loop space of a connected sum, J. London Math. Soc. (2) 64 (2001) 205-228.

[19] G.P. Paternain, Geodesic flows, Progress in Mathematics, 180 Birkäuser 1999.

[20] G.P. Paternain, J. Petean, Minimal entropy and collapsing with curvature bounded from below, Invent. Math. 151 (2003) 415-450.

[21] G.P. Paternain, J. Petean, Entropy and collapsing of compact complex surfaces, to appear in the Proceedings of the London Math. Soc.

[22] G.P. Paternain, J. Petean, Zero entropy and bounded topology, preprint 2004.

[23] J.P. Serre, Trees, Springer-Verlag 1980. 
Department of Pure Mathematics and Mathematical Statistics, University of Cambridge, Cambridge CB3 0WB, England

E-mail address: g.p.paternain@dpmms.cam.ac.uk

Cimat, A.P. 402, 36000, Guanajuato. Gto., MÉxico.

E-mail address: jimmy@cimat.mx 